\title{
Photoelectron spectroscopy at the graphene-liquid interface reveals the electronic structure of an electrodeposited cobalt/graphene electrocatalyst
}

\author{
Juan J. Velasco-Velez ${ }^{*[a, b]}$, Verena Pfeifer ${ }^{[b]}$, Michael Hävecker ${ }^{[a, c]}$, Robert S. Weatherup ${ }^{[\mathrm{d}, \mathrm{e}]}$, Rosa \\ Arrigo $^{[f]}$, Cheng-Hao Chuang ${ }^{[g]}$, Eugen Stot ${ }^{[b]}$, Gisela Weinberg ${ }^{[b]}$, Miquel Salmeron ${ }^{[e]}$, Robert \\ Schlögl ${ }^{[a, b]}$ and Axel Knop-Gericke ${ }^{[b]}$.
}

\begin{abstract}
Electrochemically grown cobalt on graphene exhibits exceptional performance as a catalyst for oxygen evolution reactions and provides the possibility of controlling the morphology and the chemical properties during deposition. However, the detailed atomic structure of this hybrid material is not well understood. To elucidate the Co/graphene electronic structure we have developed a flow cell closed by a graphene membrane that provides electronic and chemical information of active surfaces under atmospheric pressure and in the presence of liquids by means of X-ray photoelectron spectroscopy (XPS). We found that cobalt anchors on graphene via carbonyl-like species, i.e. $\mathrm{Co}(\mathrm{CO})_{\times}$promoting the reduction of $\mathrm{Co}^{3+}$ to $\mathrm{Co}^{2+}$, which is believed to be the active site of the catalyst.
\end{abstract}

Main Text: Developing new clean energy storage systems has become one of the most important challenges ${ }^{[1]}$. Electrolysis of water to produce hydrogen as a storable and clean fuel offers new opportunities to progressively replace the use of fossil fuels. Nevertheless, voltages well beyond the thermodynamic potential of $1.23 \mathrm{~V}$ are typically required to split water, mainly due to the slow kinetics of the oxygen evolution reaction (OER) at the anode ${ }^{[2]}$. To achieve a desirable current density, of at least $10 \mathrm{~mA} / \mathrm{cm}^{2}$, several hundred millivolts above the standard reaction potential are needed ${ }^{[3]}$ leading to poor process efficiencies. Metal oxides such as IrO $_{2}$ and $\mathrm{RuO}_{2}$ and compounds thereof are the most active materials under OER ${ }^{[4]}$ but these elements are expensive and listed among the rarest. Therefore, new catalysts based on abundant metal oxides and $C$ based materials have been developed ${ }^{[5]}$. Graphene has been found to be an ideal substrate for a wide range of energy related applications ${ }^{[6]}$, in particular for electrocatalysis, becoming catalytically active through its functionalization with different materials ${ }^{[7]}$.
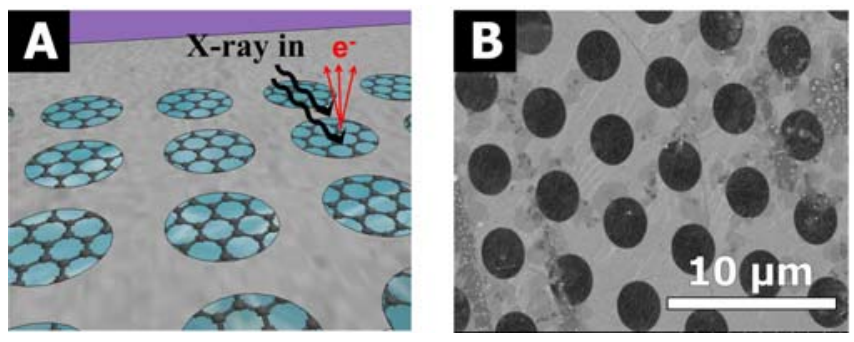

Figure 1. (A) Schematic drawing of the $\mathrm{Si}_{3} \mathrm{~N}_{4}$ grid coated by a BLG. The incoming X-ray produces two kinds of signals: photons from the core-hole decay and Auger/photo-electrons. (B) SEM image of a $\mathrm{Si}_{3} \mathrm{~N}_{4}$ grid with an array covered by BLG.

One noteworthy case is nanoscale Co grown on graphene. This system has demonstrated a remarkable performance for OER and for oxygen reduction reactions (ORR) ${ }^{[8]}$, even better than that of $C$ based electrodes functionalized with noble metal catalysts such as $\mathrm{Pt}^{[9]}$ or $\mathrm{Ir}^{[10]}$. The functionalization of $\mathrm{C}$ with low-cost metals using electrochemical procedures opens up the possibility of controlling the morphology and chemical properties of the electrodeposited metal to increase its activity, selectivity and corrosion resistance ${ }^{[11]}$. So far, the atomic structure and interaction between Co and graphene as well as the nature of the active sites, are not well understood. At present the lack of experimental methods capable of providing atomic level information about the electrochemical processes occurring at solid/liquid interfaces remains a major obstacle to the improvement of these catalysts.

Advances such as (near) ambient pressure photoelectron spectroscopy (NAP-XPS) ${ }^{[12]}$ have enabled the study of liquids under vapor pressures in the mbar range compared to previous studies in UHV. Recently, electrochemical cells based on a proton exchange membrane have been developed ${ }^{[13]}$ making it possible to investigate the electronic structure of Pt and Ir anodes during the OER under low water vapor pressures. Another promising approach combines X-ray absorption spectroscopy (XAS) in the total electron yield mode (TEY) with a frequency discrimination method using X-ray beam intensity modulation and lock-in-amplifier detection allowing the investigation of the structure of water close to a gold surface under applied bias ${ }^{[14]}$. Of late, a method based on the preparation of nanometer-thin liquid films at the tail of a wetting meniscus formed in an electrode partially immersed in the electrolyte has been developed ${ }^{[15]}$, which requires X-rays of several keV to allow photoelectrons to escape through the thin liquid film. 


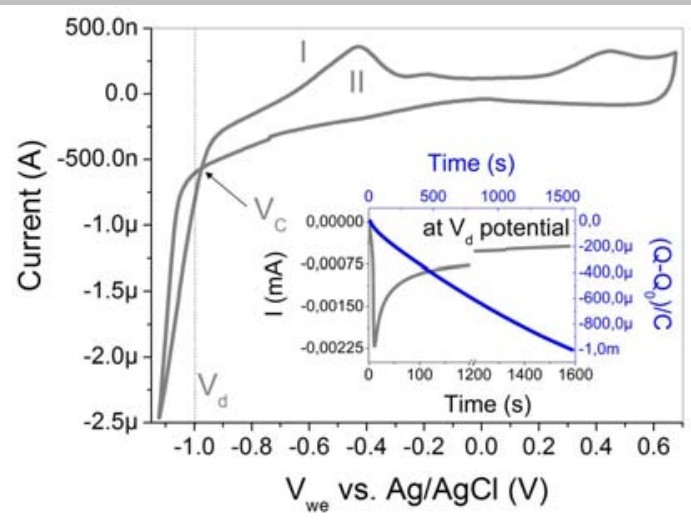

Figure 2. Cyclic voltammogram and charge transfer curve (inset) at $-1 \mathrm{~V}$ in $4 \mathrm{mM} \mathrm{CoSO}_{4}$ electrolyte.

A different approach entails the use of an electron transparent membrane based on graphene to separate the vacuum measurement chamber from a cell filled with liquid ${ }^{[16]}$. The X-rays can easily penetrate the mono- or bilayer graphene film and photoelectrons can escape and be detected on the vacuum side. Based on this idea we constructed an electrochemical liquid flow cell using a bilayer graphene (BLG) membrane following the procedure described in the supplementary information ${ }^{[17]}$. Figure 1 shows a schematic drawing of the grid of holes coated with graphene and a corresponding scanning electron microscopy (SEM) image (the full membrane is shown in figure S2). This cell allows us to detect photo- and Auger electrons generated near the solid/liquid interface and XAS in TEY and in fluorescence yield (FY) modes. The liquid flow cell (figure S3) is operated inside the main chamber of the ISISS $^{[18]}$ endstation at BESSY II at a pressure of $\sim 10^{-7}$ mbar, while aqueous solutions circulate on the back side of the membrane. Technical cell details are described in the supplementary information.

To produce catalytically active Co/graphene composites we flowed a $4 \mathrm{mM} \mathrm{CoSO}$ solution prepared with ultrapure Milli-Q water inside our cell and performed an underpotential deposition as described in figure 2. This method allows the electrochemical control of the deposition rate and the oxidation state. The complex reaction process involves several steps, such as diffusion of electroactive species, de-solvation, formation and incorporation of ad-atoms at lattice sites of the growing deposit.
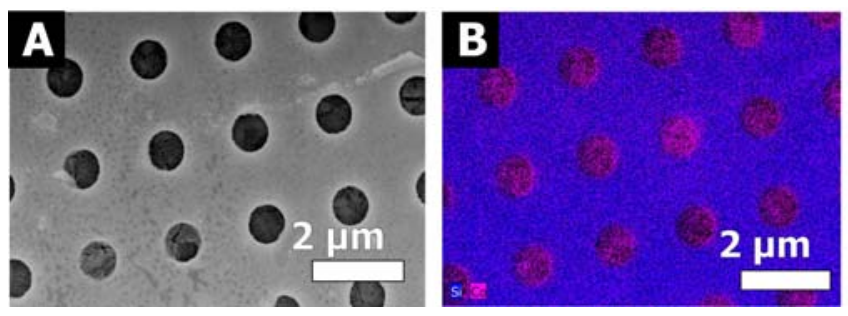

Figure 3. (A) SEM image of membrane and (B) Co/Si EDX mapping on the back side, UHV interface (Co is magenta and Si is blue).

Figure 2 shows a cyclic voltammogram (CV) recorded at $20 \mathrm{mV} / \mathrm{s}$. The cross-potential $\left(\mathrm{V}_{\mathrm{C}}\right)$ at $-0.9 \mathrm{~V}$ corresponds to the equilibrium potential of the metal redox couple $\left(\mathrm{Co}^{2+} / \mathrm{Co}^{0}\right)^{[19]}$. Peaks I and II are associated with the dissolution of deposited Co or with the oxidation of two different Co phases ${ }^{[20]}$. The inset of figure 2 shows a chronoamperogram (CA) and the total charge transferred during electrodeposition at a deposition voltage $\left(\mathrm{V}_{\mathrm{d}}\right)$ of $-1.0 \mathrm{~V}$. The total charge transferred was $1 \mathrm{mC}$ after $\sim 1600 \mathrm{~s}$. Given this, the thickness of the electrodeposited Co can be estimated from the equation:

$$
x=\frac{M \cdot Q_{T}}{\rho \cdot A \cdot n \cdot F},
$$

where $\mathrm{M}=$ molar mass, $\mathrm{Q}_{\mathrm{T}}=$ total charge transferred, $\rho=$ density, $\mathrm{A}=$ effective area, $\mathrm{n}=$ valence of the metal, and $\mathrm{F}=\mathrm{Faraday}$ 's constant. The electrodeposited film was probed with SEM and energy dispersive X-ray spectroscopy (EDX) from the side exposed to UHV (see figure 3). The images show that Co (magenta) has been deposited across the graphene membrane as all graphene-covered holes show a relatively homogeneous Co coverage.

The OER activity of the Co electrodeposited onto graphene was evaluated with a $10 \mathrm{mM} \mathrm{KOH}$ solution. Figure 4 a shows the CVs (recorded at $20 \mathrm{mV} / \mathrm{s}$ ) of the pristine graphene and the Co/graphene electrodes. For the electrodeposited Co the CV shows two oxidation peaks and one reduction peak ${ }^{[21]}$. The anodic peaks observed at $\sim 0.6 \mathrm{~V}$ (I) and $\sim 1.2 \mathrm{~V}$ (II) are ascribed to the oxidation of $\mathrm{Co}^{2+}$ to $\mathrm{Co}^{3+}$ and $\mathrm{Co}^{3+}$ to $\mathrm{Co}^{4+}$, respectively. The peak at $\sim 1.1 \mathrm{~V}$ (III) is assigned to the transition from $\mathrm{Co}^{4+}$ to $\mathrm{Co}^{2+}$ when the potential is reversed indicating that the OER is preceded by Co oxidation. In addition the OER was analyzed by linear sweep voltammetry (LSV) at a scan rate of $20 \mathrm{mV} / \mathrm{s}$ (see figure $4 \mathrm{~b}$ ). A noteworthy overpotential decrease due to the presence of the Co is seen, which was evaluated at a current density of $10 \mathrm{~mA} / \mathrm{cm}^{2}$. 

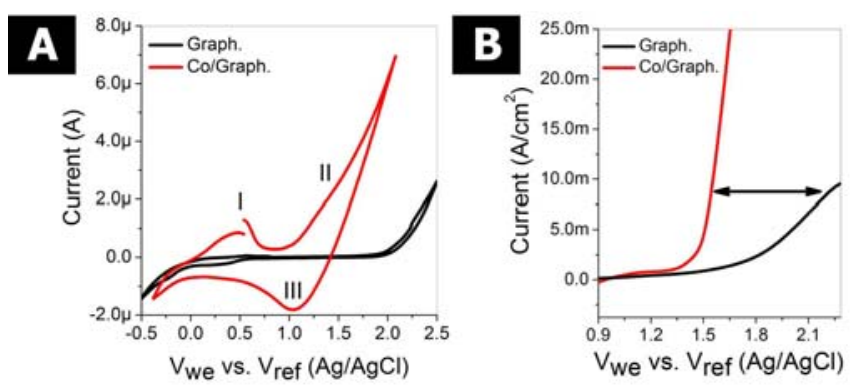

Figure 4. (A) $\mathrm{C}$ and (B) LSV of pristine graphene and electrodeposited Co on graphene. The measurements were performed under 10 mM of $\mathrm{KOH}$ using a three electrode cell with $\mathrm{Pt}$ counter and $\mathrm{Ag} / \mathrm{AgCl}$ reference electrodes.

The electronic structure of the electrodeposited Co onto graphene was investigated by means of X-ray spectroscopy. Accordingly, the in situ XAS measurements were conducted under operating conditions. The $C_{0} L_{3,2}$-edges involve excitations of the $2 p$ electrons to the unoccupied Co $3 \mathrm{~d}$ states and are sensitive to the valence and the coordination environment of the Co atoms ${ }^{[22]}$. Figure $5 \mathrm{a}$ shows XA spectra recorded before and after the electrodeposition of Co, which were recorded in TEY mode (lower panel, red spectra) from the photoelectrons collected by the graphene membrane and in FY mode (top panel, green spectra) from the photons that escape through the membrane. The TEY mode is more sensitive to the layers near the Co/graphene interface due to the short inelastic mean free path of electrons in solids while the FY is mostly bulk sensitive because of the larger photon penetration depth. Thus, the combination of these two modes provides insights concerning the anchoring between Co and graphene as well as details of the bulk chemical state. After the electrodeposition, the TEY signal for the Co-L edge is consistent with the rock salt CoO structure with an intense peak at $777.4 \mathrm{eV}$ associated with $\mathrm{Co}^{2+[23]}$. Meanwhile, the $\mathrm{FY}$ spectrum is characteristic of $\mathrm{Co}_{3} \mathrm{O}_{4}$, as indicated by the intense $\mathrm{Co}^{3+}$ peak at $780.2 \mathrm{eV}$. Consequently, the anchoring of $\mathrm{Co}$ to graphene prompts the reduction from $\mathrm{Co}^{3+}$ (bulk) to $\mathrm{Co}^{2+}$ (interface), which is associated with the substitution of $\mathrm{O}$ ligands by $\mathrm{C}$ in $\mathrm{Co}_{3} \mathrm{O}_{4}$ in the form of $\mathrm{Co}(\mathrm{CO})_{\mathrm{x}}$.

In addition, the electronic structure was investigated with XPS, which probes the core level binding energies of the constituent species. Figure $5 b$ shows the Co $2 p$ XPS region collected before and after the Co electroplating process. The Co $2 p$ spectra feature a doublet $2 p_{3 / 2}$ and $2 p_{1 / 2}$ with a spin orbit splitting $\Delta E$ of $15.5 \mathrm{eV}$. Peak assignment and species quantification is challenging as most of the species appear within a $2.5 \mathrm{eV}$ binding energy $(\mathrm{BE})$ range, including the complex satellite structure characteristic of first row transition metals ${ }^{[24]}$, which is indicative of $\mathrm{Co}^{2+} / \mathrm{Co}^{3+}$ with unpaired $\mathrm{d}$ electrons and could be associated to an oxygen rich environment. Therefore, some ambiguity in the peak assignment and quantification of such components still exists due to species with similar BE resulting in overlapping peaks of adjacent species ${ }^{[25]}$. It has been suggested that the XPS $2 p$ peak deconvolution of transition metal species cannot be done using a single peak approach due to multiplet splitting and satellite structure ${ }^{[26]}$. The close interaction of $C$ with Co can be compared to a previous XPS investigation of pristine and deposited cobaltocene films ${ }^{[27]}$. In this investigation, the pure cobaltocene film showed two main peaks at binding energies of $\sim 783 \mathrm{eV}$ and $\sim 798 \mathrm{eV}$ corresponding to the spin-orbit split Co $2 \mathrm{p}_{3 / 2}$ and Co $2 p_{1 / 2}$ components in good agreement with our measurements. Accordingly, the Co $2 p_{3 / 2}$ spectrum here is deconvoluted using two dominant Gaussian-Lorentzian peaks at binding energies of $783.2 \mathrm{eV}$ (Peak A) and $785.6 \mathrm{eV}$ (Peak B) with two shake-up satellites at $6 \mathrm{eV}$ higher $\mathrm{BE}$ than the main peaks $\left(\mathrm{S}_{\mathrm{A}}\right.$ and $\left.\mathrm{S}_{\mathrm{B}}\right)$. The $\mathrm{Co}_{0} \mathrm{p}_{3 / 2} / \mathrm{Co}_{2} \mathrm{p}_{1 / 2}$ intensity ratio was fixed to 2 . The peak at $\sim 783.2$ $\mathrm{eV}($ Peak $\mathrm{A})$ is typically attributed to $\mathrm{Co}^{2+[28]}$. Therefore, $\mathrm{Co}^{2+}$ bound to $\mathrm{C}$ appears responsible for the peak observed here at this same $\mathrm{BE}$, which shows multiplet splitting and satellite structure resulting in the majority of the signal seen. We note that minor amounts of $\mathrm{Co}_{x} \mathrm{O}_{y}(\mathrm{OH})_{z}$ may be present and contribute to the Co $2 \mathrm{p}$ spectrum ${ }^{[29]}$. Furthermore, the peaks at $783.2 \mathrm{eV}$ (Peak $\left.\mathrm{A}\right)$ and $785.6 \mathrm{eV}$ (Peak B) can also be related to the formation of Co bound to oxygen in the form of carbonyl-like species such as $\mathrm{Co}(\mathrm{CO})_{x}^{[24,30]}$ as comparison with pyrolyzed and porphyrin-Co samples reveals. The higher binding energy side of these peaks overlap with the shake-up satellite envelopes $S_{A}$ and $S_{B},{ }^{[26,31]}$ which are associated with the presence of $\mathrm{Co}^{2+}$ species. Consequently, the synergistic interaction between $\mathrm{Co}$ and graphene is probed, which reveals that the anchoring of $\mathrm{Co}$ to the graphene by means of $\mathrm{Co}(\mathrm{CO})_{\times}$bonds yields the formation of $\mathrm{Co}^{2+}$ species.
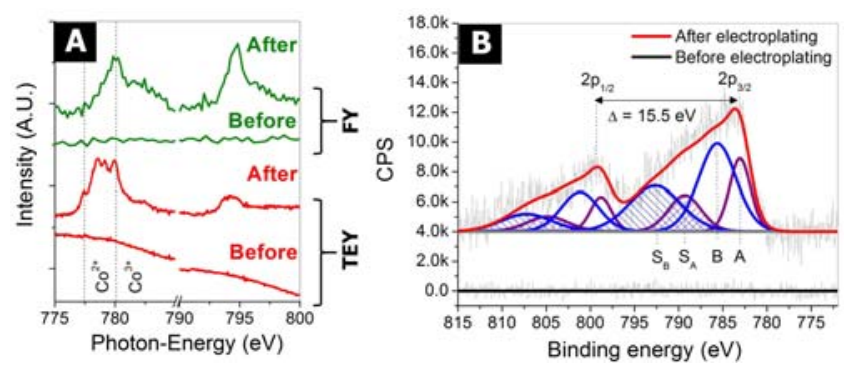

Figure 5. A) XAS Co L-edge collected in TEY mode (lower panel, red spectra) before and after electroplating, and FY (upper panel, green spectra). (B) Co 2p XPS region before (bottom, black curve) and after the electroplating (top, red curve).

In summary the anchoring and chemical state of electrodeposited Co on a graphene electrode and its OER electrochemical activity was investigated under operando conditions. This analysis is made feasible by the development of a novel electrochemical cell incorporating an electron transparent membrane based on BLG that facilitates electron spectroscopy from electrode-electrolyte interfaces during potentiometric control. Using this approach, we demonstrated that the electrodeposition of Co onto graphene gives rise to the reduction of $\mathrm{Co}^{3+}$ to $\mathrm{Co}^{2+}$ species at the interface. The anchoring of Co to graphene is due to the formation of Co bound to oxygen in the form of $\mathrm{Co}(\mathrm{CO})_{x}$ species. Therefore, the enhancements in electrocatalytic activity and stability under OER shown by 
Co/Graphene composites are likely related to the hybrid interface contacts, which control and promote electron transfer reactions The increase in the catalytic activity as well as the binding mechanism of the Co oxide catalyst is attributed to $\mathrm{Co}^{2+}$ active sites derived from the reduction of $\mathrm{Co}^{3+}$ species. This novel setup opens a way for studies of electrode processes with high sensitivity to the interfaces both in surface sensitive electron spectroscopy and in electron microscopy based techniques.

\section{Acknowledgements}

We thank to the staff at BESSY II of the HZB for operational support. HZB is acknowledged for granting beamtime at the ISSIS endstation under proposal \#14201159. This work was funded from EU project GRAFOL grant 285275 and by SYNESTESia project grant 05K14EWA (BMBF). R.S.W. acknowledges a Research Fellowship from St. John's College, Cambridge and a Marie Skłodowska-Curie Individual Fellowship (Global) under grant ARTIST (no. 656870) from the European Union's Horizon 2020 research and innovation programme.. C.H.C. acknowledges financial support from projects 103-2112-M-032-004 and 102-2632-M-032-001 -MY3. M.S. was supported by the Office of Science, Division of Materials Sciences and Engineering, of the U.S. Department of Energy (DOE) under Contract No. DE-AC02-05CH11231. Philipp Braeuninger-Weimer is gratefully acknowledged for providing CVD graphene on Cu foil and Stephan Hofmann for useful discussions.

Keywords: Photoelectron spectroscopy• X-ray absorption spectroscopy• Aqueous conditions • Graphene/Cobalt• Electrodeposition

[1] J. Chow, R. J. Kopp, P. R. Portney, Science 2003, 302(5650), 1528-1531.

[2] M. G. Walter, E. L. Warren, J. R. McKone, S. W. Boettcher, Q. Mi, E. A. Santori, N. S. Lewis, Chem. Rev. 2010, 110(11), 6446-6473.

[3] a) K. Gong, F. Du, Z. Xia, M. Durstock, L. Dai, Science 2009, 323(5915), 760-764; b) Y. Gorlin, T. F. Jaramillo, J. AM. Chem. Soc. 2010, 132(39), $13612-13614$

[4] a) Y. Lee, J. Suntivich, K. J. May, E. E. Perry, Y. Shao-Horn, J. Phys. Chem. Lett. 2012, 3(3), 399-404; b) T. C. Wen, C. C. Hu, J. Electrochem. Soc. 1992, 139(8), 2158-2163.

[5] a) I.V. Lightcap, T. H, Kosel, P. V. Kamat, Nano Lett. 2010, 10(2), 577-583; b) P.V. Kamat, J. of Phys. Chem. Lett. 2009, 1(2), 520-527.

[6] a) S. Bai, X. Shen, RSC Adv. 2012, 2(1), 64-98; b) B. F. Machado, P. Serp, Catal. Sci. Technol. 2012, 2(1), 54-75; c) S. Bose, T. Kuila, A. K. Mishra, R. Rajasekar, N. H. Kim, J. H. Lee, J. Mater. Chem. 2012, 22(3), 767-784.

[7] a) Z. S. Wu., G. Zhou, L. C. Yin, W. Ren, F. Li, H. M. Cheng, Nano Energy 2012, 1(1), 107-131; b) Y. Liang, Y. Li, H. Wang, J. Zhou, J. Wang, T. Regier, H. Dai, Nature Mater 2011, 10(10), 780-786.

[8] S. Mao, Z. Wen, T. Huang, Y. Hou, J. Cheng, Ener. \& Environ. Sci. 2014, 7(2), 609-616

[9] J. Wang, J. Zhou, Y. Hu, T. Regier, Ener. Environ. Sci. 2013, 6(3), 926-934.

[10] L. Wu, C. H. Wu, H. Zhu, A. Mendoza-Garcia, B. Shen, J. Guo, S. Sun, JACS 2015, 137(22), 7071-7074.

[11] B. Bozzini, P. Bocchetta, A. Gionancelli, C. Mele, M. Kiskinova, Acta Chimica Slovenica 2014, 61(2), $263-271$.

[12] M. Salmeron, R. Schlögl, Surf. Sci. Rep. 2008, 63(4), 169-199; b) D.E. Starr, Z. Liu, M. Hävecker, A. Knop-Gericke, H. Bluhm, Chem. Soc. Rev. 2013, 42(13), 5833-5857.

[13] a) R. Arrigo, M. E. Schuster, C. Ranjan, E. Stotz, A. Knop-Gericke, R. Schlögl, Angew. Chem. Int. 2013, 52(44), 11660-11664; b) H. S. Casalongue., S. Kaya, V. Viswanathan, D. J. Miller, D. Friebel, H. A. Hansen, J. K Nørskov, A. Nilsson, H. Ogasawara, Nature Comm. 2013, 4;C) H. S. Casalongue, M. L. Ng, S. Kaya, D. Friebel, H. Ogasawara, A, Nilsson, Angew. Chem. Int. 2014, 126(28), 7297-7300.

[14] J.J. Velasco-Velez, C. H. Wu, T. A. Pascal, L. F. Wan, J. Guo, D. Prendergast, M. Salmeron, Science 2014, 346(6211), 831-834

[15] a) O. Karslioglu et al., Faraday Discuss. 2015; b) S. Nemšak et al. Nature commun. 2014, 5, 5441

[16] A. Kolmakov, D. A. Dikin, L. J. Cote, J. Huang, M. K. Abyaneh, M. Amati, L. Gregoratti, S. Günter, M. Kiskinova, Nature Nanotechnol. $2011,6(10)$, 651-657.

[17] a) R. S. Weatherup, B. Dlubak, S. Hofmann, ACS Nano 2012, 6, 9996-10003; b) P. R. Kidambi, C. Ducati, B. Dlubak, D. Gardiner, R. S. Weatherup, M. B. Martin, P. Seneor, H. Coles, S. Hofmann, J. Phys. Chem. C 2012, 116, 22492-22501; c) J. J. Velasco-Velez, C. H. Wu, B. Y. Wang, Y. Sun, Y. Zhang, J. H. Guo, M. Salmeron, J. Phys. Chem. C 2014, 118(44), 25456-25459; d) J. J. Velasco-Velez, C. H. Chuang, H. L. Han, I. Martinez-Fernandez, C. Martinez, W. F. Pong, Y. R. Shen, F. Wang, Y. Zhang, J. Guo, M. Salmeron, J. of the Electrochem. Soc. 2013, 160(9), C445-C450; e) S. Hofmann, P. Braeuninger-Weimer, and R.S. Weatherup, J. Phys. Chem. Lett. 2015, 6, 2714-2721.

[18] A. Knop-Gericke et al., Advances in Catalysis 2009, 52, 213-272.

[19] S. Fletcher, Electrochemi. Acta 1983, 28(7), 817-923.

[20] M. Palomar-Pardave, I. Gonzalez, A. B. Soto, E. M. Arce, Electroanal. Chem. 1998, 443(1), 125-136.

[21] J. Ismail, M. F. Ahmed, P. V. Kamath, J. Power Sourc. 1991, 36(4), 507-516.

[22] Y. Liang et al., J. Am. Chem. Soc. 2012, 134(38), 15849-15857.

[23] Q. He, Q. Li, S. Khene, X. Ren, F. E. Lopez-Suarez, D. Lozano-Castello, A. Bueno-Lopez, G. Wu, J. Phys. Chem. C 2013, 117(7), 8697-8707.

[24] J. J. Pietron, J. C. Biffinger, S. B. Qadri, D. R. Rolison, J. Mater. Chem. 2011, 21(21), 7668-7677.

[25] K. Artyuskova, S. Levendosky, J. Fulghum, P. Aanassov, Topics in Cat. 2007, 46(3-4), 263-275.

[26] M. C. Biesinger, B. P: Payna, A. P. Grosvenor, L. W. M. Lau, A. R. Gerson, R. St. C. Smart, Appl. Surf. Sci. 2011, 257(7), 2717-2730.

[27] C. K. Chan, A. Kahn, Q. Zhang, S. Barlow, S. R. Marder, Appl. Phys. 2007, 102(1), 014906.

[28] a) X. Xiang, L. Zhang, H. I. Hima, F. Li, D. G. Evans, Appl. Clay. Sci. 2009, 42(3), 405-409. b) V. Musat, E. Fortunato, A. M. B. Do Rego, R. Monteiro, Thin Solid Films 2008, 516(7), 1499-1502; c) R. S. Da Cruz, A. J. S. Mascarenhas, H. M. C. Andrade, Appl. Cat. B: Environ. 1997, $18(3)$, $223-231$.

[29] a) S. Cobo et al. Nature Mat. 2012, 11(9), 802-807; b) R. Xu, H. C. Zeng, Chem. Mat. 2003, 15(10), 2040-2048.

[30] a) K. Artyushkova, S. Pylypenko, T. S. Olson, J. E. Fulghum, P. Atanasov, Langmuir 2008, 24(16), 9082-9088; b) S. Pylypenko, S. Mukherjee, T. S. Olson, P. Atanassov, Electrochim. Acta 2008, 53(27), 7875-7883; c) T. S. Olson, S. Pylypenko, P. Atanassov, K. Yamada, H. Tanaka, J. Phys. Chem. C 2010, 114(11), 5049-5059.

[31] M. Hu, Y. Murakami, M. Ogura, S. Maruyama, T. Okubo, J. Catal 2004, 225(1), 230-239. 
[a] Dr. J. J. Velasco-Velez, Dr. M, Hävecker, Prof. Dr. R. Schlög

Max Planck Institute for Chemical Energy Conversion

Mülheim 45470, Germany

*E-mail: velasco@fhi-berlin.mpg.de

[b] Dr. J. J. Velasco-Velez, V. Pfeifer, E. Stotz, G. Weinberg, Prof. Dr. R. Schlögl, Dr. A. Knop-Gericke

Fritz Haber Institute der Max-Planck-Gesellschaft

Berlin 14195, Germany

[c] Dr. M. Hävecker

Helmholtz-Center Berlin for materials and Energy, BESSY II

Berlin 12489, Germany

[d] Dr. R. S. Weatherup

Engineering Department, University of Cambridge

Cambridge CB3 OFA, United Kingdom

[e] Dr. R. S. Weatherup, Prof. M. Salmeron

Materials Science Division

Lawrence Berkeley National Laboratory

Berkeley 94720, USA

[f] Dr. R. Arrigo

Diamond Light Source

Oxfordshire 0X11 0QX, United Kingdom

[g] Prof. Dr. C-H. Chuang

Department of Physics

Tamkang University

Tamsui 251, Taiwan

Supporting information for this article is given via a link at the end of the document. 